\title{
Tomographic analysis of the De Sitter model in quantum and classical cosmology
}

\author{
C. Stornaiolo \\ ${ }^{3}$ Istituto Nazionale di Fisica Nucleare, \\ Sezione di Napoli, \\ Complesso Universitario di Monte S. Angelo \\ Edificio 6, via Cinthia, 45 - 80126 Napoli, Italy
}

June 25, 2021

\begin{abstract}
The importance of the tomographic approach is that either in quantum mechanics as in classical mechanics the state of a physical system is expressed with the same family of functions, the tomograms. The extension of this procedure to quantum cosmology is straightforward. But instead of using the tomographic representation, we use tomograms to analyze the properties of the quantum and classical universes, starting from the wave functions in quantum cosmology and the phase space distribution in classical cosmology. In this paper we resume the properties of the tomographic approach introduced in previous papers. Then we study and discuss the properties of the initial conditions introduced by Hartle and Hawking and by Vilenkin and Linde and we study their classical transition. It results that a possible reason for the quantum to classical transition is the decay of the cosmological constant from the Planck scale to the present one. So that the Cosmological Constant Problem becomes a crucial topic in study of the evolution of the universe.
\end{abstract}

\section{Introduction}

General Relativity is a well-established theory which introduces a close relation between geometry and physics. However its undeniable successes are in some cases counterbalanced by some of its shortcomings.

The Hawking and Penrose theorems show that under conditions of the energy-momentum tensor, which are compatible with alle the forms of matter fields we know, the solutions of the Einstein equations must 
contain at least one singularity. This means that the theory is not always able to make physical predictions because the presence a singularity means that the state of a gravitational system can not determined. Because as the state of a physical system is represented by a set of data that allow us to predict its evolution. The presence of a singularity means the absence of such data and therefore the impossibility to predict the physical evolution of the system.

In cosmology the singularity is related to the degeneracy of the metric and of the scalar curvature and it corresponds to an infinite massenergy density. This suggests that at states close to the singularity General Relativity can not be a reliable theory.

The high densities and small distances involved suggest that it is most likely to be a quantum theory that has general relativity as its classical limit.

In the quest of this theory we can apply the canonical quantization of the gravitational field, according to the prescriptions introduced by Dirac in 1932. This program was completed by Arnowitt, Deser and Misner 1 by introducing the Hamitonian formulation of general relativity where the spatial components of the metric and their respective conjugate momenta defined on a spatial 3-surface $S$ are the canonical variables. The Einstein equations are equivalent to two classes of equations, the evolution equation obtained the Hamiltonian equation of motion, but the properties of the theory imply too the existence of constraint equations among the variables which, according to the Bianchi identities, are preserved during the evolution of the system on a each spatial 3-surface $S$.

In quantum gravity the role of the Schroedinger equations is taken by the quantum constraint equations (the Wheeler De Witt equation and the diffoemorphism equation), which become operators that annihilate the wave functionals. A direct development of this formalismo is loop quantum gravity that will be discussed elsewhere.

A relevant chapter of quantum gravity is quantum cosmology. It is considered as a simplified version of quantum gravity because it takes as canonical variables only homogeneous spatial metrics and there conjugate momenta. This restriction reduces the infinite degrees of freedom of the quantum gravity theory to a finite number, by this simplification many properties of the general theory can be explored with more ease. But the study of quantum cosmology has become an important issue in modern physics because it can explain the origin of the large structure of the universe.

The two fundamental laws at the basis of quantum cosmology theory are the dynamical description of the quantum evolution of the universe and the assignment of the initial quantum state.

The Wheeler-DeWitt equation equation in quantum cosmology plays a role similar to the Schrödinger equation in quantum mechanics.

Among the infinite solutions of the Wheeler-De Witt equation the 
physical ones are those which have a classical limit and predict the large scale of the universe observed. . According to our present knowledge we live in a almost homogeneouos and isotropic universe, very likely flat, whose particular structure was originated by primordial quantum perturbations generated during the inflationary epoch. The acceleration of the observed expansion recently completes the current picture of the state of the universe. The origin of this expansion has not yet been definitively explained and is presumably attributable either to a form of dark energy or to a substantial modification of Einstein's equations.

This scenario must be the result of the evolution of a classical primordial universe descending from a quantum universe whose properties are in turn determined by particular initial conditions. Such initial conditions must be regarded as a fundamental physical law, because unlike an ordinary physical system they can not be arbitrarily assigned in relation to the conditions designed by an observer. They must select the only quantum state that conducts to the correct evolution of the universe.

The choice of the initial state of the universe can be deduced from particular physical requirements, first imposing the absence of an initial singularity and second by requiring that the collapsing modes will not make the newborn universe recollapses. Alternatively one can also think that some informations of the initial stages of the universe survive on the present structure and that they can be iderived from observational data.

In this article we will discuss the initial conditions proposed by Hartle and Hawking, Vilenkin and Linde, in particular we consider them, for sake of simplicity, in the context of a de Sitter, i. e. just in presence of a cosmological constant. We analyze the different solutions in the context of quantum tomography. It is interesting to note that greater versatility is obtained in the understanding of an argument, when the different representations of quantum mechanics are used simultaneously.

Quantum tomography was introduced in very recent times to have a means to reconstruct the initial state of a quantum system by detecting probability distribution functions. An observer gets a statistical prediction of the values of any observable as the result of the measurements within the limits imposed by quantum mechanics.

To this purpose q uantum tomography has been applied quantum optics by using the properties of balanced homodyne detection [4] [5] [6]. The homodyne measurement of an electromagnetic field gives all the possible linear combinations of the field quadratures just by varying the phase of a local oscillator. At any phase of the local oscillator the average of the outcomes is related to a marginal distribution of the Wigner function [7]. This is the most straightforward way to reconstruct experimentally the Wigner function and consequently any other quasi probabilistic distribution.

Similarly quantum tomography is applied to quantum mechanics to 
mount an atomic optics apparatus to reconstruct the initial state of ta quantum system (see [8] for the problem in one dimension and [9] for the problem in two dimensions) . The experiment was realized by [10 in reconstructing the initial state of a helium atom(see also [11 for the realization of the detector).

Theoretical research has shown that conventional quantum mechanics can have a representation in which the fundamental quantities describing the quantum states are the tomograms instead of the wave function. In many respects it is useful the tomographic representation cf quantum mechanics, because tomograms have an immediate physical interpretation because they are marginal probability functions therefore they are observables. The other important aspect is that one can define classical tomograms on the phase space and we can describe the physical state of a system in a single way both in quantum mechanics and in classical mechanics.

We can to extend quantum tomography to cosmology. It seems an appropriate way to address the issue of the initial conditions problem. We will show how to construct quantum and classical tomograms, study their properties and then consider the transition from quantum to classical, in order to try to connect the properties of the large scale universe with the initial conditions.

In sect. 2 symplectic tomography is introduced. We introduce the definition of a quantum tomogram as the Radon transform of the Wigner function. From this we find also the relation of tomogram and wave function. In the following are listed all the properties of the tomograms and how to calculate them with the stationary phaase approximation. The definition of classical tomograms ends the section.

Classical cosmology is introduced in sect. 3. After a very short resume of the relevant properties of the de Sitter universe, we derive the tomogram after defining the probability distribution on the phase space through the Hamiltonian classical contraint. In sect.4 we consider the wave equations corresponding to the proposals of Hartle-Hawking, Vilennkin and Linde as solutions of the Airy equations. But only in the first case it is possible to express the Wigner function as an Airy function as well. The other two finctions are not limited so it is not possible to perform an integral transform. The same is happens in the definition of the tomograms.We conclude the section by taking the classical limit.The arguments developed in this paper open new perspectives in the study of quantum cosmology. In sect 6 after resuming the results of this paper, we list the possible developments of this work, which was conceived originally to relate the data on the large scale structure of the universe with the initial conditions, but it shows also the power of analysis of tomography in understanding quantum cosmology. 


\section{Introduction to symplectic tomography}

In classical mechanics the state of a physical system is given by that set of data that allow us to predict the evolution of this system on the basis of the physical laws that rule them.

For example, the initial position and velocity of a point particle in a potential allows to determine its the motion, which in turn is described as a succession of states determined uniquely by the equation of motion.

In phase space the state of a single classical point particle is given by the distribution function

$$
f(q, p)=\delta\left(q-q_{0}\right) \delta\left(p-p_{0}\right) .
$$

Due to the uncertainty principle according to which it is not possible to fix with arbitrarily high precision the position and the momentum of a particle in the same time, this definition of physical state does not make sense in quantum mechanics. Rather the state of a system is intended in terms of a probability distribution for each observable.

According to [12] there are nine formulations of quantum mechanics, the tomographic representation is the tenth. In each of these formulations the physical state of a quantum system is represented differently.

For example Wigner introduced a function on phase space defined by the transform of the wave function $\psi(x)$

$$
W(x, p)=\frac{1}{2 \pi} \int \psi\left(x+\frac{\hbar u}{2}\right) \psi^{*}\left(x-\frac{\hbar u}{2}\right) \mathrm{e}^{-i u p} d u .
$$

to describe the state of a quantum particle on the phase space in analogy with classical mechanics. The Wigner function is defined with the requirement that it has to be normalized

$$
\int W(q, p) d q d p=1 \text {. }
$$

In quantum cosmology alongside the concept of wave function of the universe, the density matrix representation and the phase space representations have been introduced with the purpose of addressing the problem of the classical transition for a quantum universe.

Unfortunately, these formalisms can hardly be directly related to the observational data, because the wave function and the density matrix do not have a physical interpretation, while the Wigner function cannot be considered a probability function as it can take negative values unlike the Boltzmann which instead is probability distribution function on phase space,

Recently the notion of standard positive probability distribution function (tomogram) was introduced in cosmology to describe the quantum state of universe as an alternative to wave function or to density matrix 
The tomogram can be defined by projecting the Wigner function on the axes on the phase space at arbitrary angles. Mathematically this is represented by the modified Radon transform,

$$
\mathcal{W}(X, \mu, \nu)=\int W(q, p) \delta(X-\mu x-\nu p) d x d p .
$$

The function $\mathcal{W}(X, \mu, \nu)$ depends on the random variable $X$ and two real parameters $\mu$ and $\nu$. Specifically $X$ is given by a linear combination of position and momentum:

$$
X=\mu x+\nu p
$$

with $\mu=s \cos \theta$ and $\nu=s^{-1} \sin \theta$.

So, we say that the quantum state is given if the position probability distribution $\mathcal{W}(X, \mu, \nu)$ in an ensemble of rotated and squeezed reference frames in classical phase space is given. Given a large ensemble of particles prepared in the same state, it gives the probability that the pseudo classical trajectory of a single particle depends on a pair of initial conditions contained in the combination [2.5] .

From (2.7) we can derive the relation between a wave function $\psi(x)$ and its corresponding tomogram. We must consider separately the case $\nu \neq 0$ and the case $\nu=0$.

Let us consider first $\nu \neq 0$. It is convenient to write the Wigner function in the following form

$$
W(x, p)=\frac{1}{2 \pi} \int \psi\left(y^{\prime}\right) \psi^{*}(y) \mathrm{e}^{i p\left(y^{\prime}-y\right)} d\left(y^{\prime}-y\right)
$$

so that the tomogram is written as

$$
\mathcal{W}(X, \mu, \nu)=\frac{1}{2 \pi} \int \psi(y) \psi^{*}\left(y^{\prime}\right) \mathrm{e}^{i p\left(y^{\prime}-y\right)} \delta(X-\mu x-\nu p) d\left(y^{\prime}-y\right) d x d p
$$

where

$$
\begin{aligned}
y=x-\frac{u}{2} & x=\frac{y+y^{\prime}}{2} \\
y^{\prime}=x+\frac{u}{2} & u=y^{\prime}-y .
\end{aligned}
$$

Applying the definition of tomogram we find

$$
\begin{aligned}
\mathcal{W}(X, \mu, \nu) & =\frac{1}{2 \pi} \int \psi\left(y^{\prime}\right) \psi^{*}(y) \mathrm{e}^{i p\left(y^{\prime}-y\right)} \delta(X-\mu x-\nu p) d\left(y^{\prime}-y\right) d x d p \\
& =\frac{1}{2 \pi|\nu|} \int \psi(y) \psi^{*}\left(y^{\prime}\right) \mathrm{e}^{i\left(\frac{X}{\nu}-\frac{\mu}{2 \nu}\left(y+y^{\prime}\right)\right)\left(y^{\prime}-y\right)} d\left(y^{\prime}-y\right) d x \\
& =\frac{1}{2 \pi|\nu|} \int \psi(y) \psi^{*}\left(y^{\prime}\right) \mathrm{e}^{i\left(\frac{X}{\nu}\left(y-y^{\prime}\right)-\frac{\mu}{2 \nu}\left(y^{2}-y^{\prime 2}\right)\right)} d y^{\prime} d y \\
& \equiv \frac{1}{2 \pi|\nu|}\left|\int \psi(y) \exp \left[i\left(\frac{\mu}{2 \nu} y^{2}-\frac{X}{\nu} y\right)\right]\right|^{2}
\end{aligned}
$$




$$
\begin{aligned}
& \text { If } \nu=0 \text { we have } \\
& \qquad \begin{aligned}
\mathcal{W}(X, \mu, 0) & =\frac{1}{2 \pi} \int \psi\left(x+\frac{u}{2}\right) \psi^{*}\left(x-\frac{u}{2}\right) \mathrm{e}^{i u p} \delta(X-\mu x) d x d p d u \\
& =\frac{1}{2 \pi} \int \psi\left(x+\frac{u}{2}\right) \psi^{*}\left(x-\frac{u}{2}\right) \delta(u) \delta(X-\mu x) d x d u \\
& =\frac{1}{2 \pi} \int \psi(x) \psi^{*}(x) \delta(X-\mu x) d x \\
& =\frac{1}{2 \pi|\mu|}\left|\psi\left(\frac{X}{\mu}\right)\right|^{2}=\frac{1}{2 \pi|\mu|}|\psi(x)|^{2},
\end{aligned}
\end{aligned}
$$

because with $\nu=0$

$$
X=\mu X
$$

in particular

$$
\mathcal{W}(X, 1,0)=\frac{1}{2 \pi}|\psi(X)|^{2} \equiv \frac{1}{2 \pi}|\psi(x)|^{2} .
$$

It must be noted that the general expression for the tomogram 2.10 is the square modulus of the tomographic amplitude

$$
\mathcal{A}(X, \mu, \nu)=\int \psi(y) \exp \left(i \frac{\mu y^{2}}{2 \nu}-i \frac{X y}{\nu}\right) d y
$$

which is the fractional Fourier transform of the wave function.

When $\mu=0$ and $\nu=1$ the tomographic amplitude reduces to a Fourier transform from $x$ to $X \equiv p$, leading in 2.10 to the density $|\psi(p)|^{2}$ in the $p$ representation.

The relation $X=\mu x+\nu p$ between $x, p$ and $X$ is a collection of linear canonical transformations parameterized by $\mu$ and $\nu$ with generating function

$$
G_{(\mu \nu)}(x, X)=-\frac{\mu}{2 \nu}\left(x-\frac{X}{\mu}\right)^{2}=-\frac{\mu}{2 \nu} x^{2}+\frac{X x}{\nu}-\frac{X^{2}}{2 \mu \nu}
$$

so that 2.10 can be written as

$$
\mathcal{W}(X, \mu, \nu)=\frac{1}{2 \pi|\nu|}\left|\int \psi(y) \exp \left[-i G_{(\mu \nu)}(y, X)\right] d y\right|^{2} .
$$

Eq. 2.15) can be interpreted as the square modulus of the wave function obtained by a linear canonical transform in the new variable $X$.

Finally we observe that the relation found between tomogram and wave function can be applied to the density matrix of a pure state and extended to any density matrix in the following way,

$$
\mathcal{W}(X, \mu, \nu)=\frac{1}{2 \pi|\nu|} \int \rho\left(y, y^{\prime}\right) \exp \left[-i \frac{y-y^{\prime}}{\nu}\left(X-\mu \frac{y+y^{\prime}}{2}\right)\right] d y d y^{\prime} .
$$


These relations can all be inverted to reconstruct the Wigner function, the density matrix and the wave function once the tomograms have been determined. As a matter of fact it can be proved that there exist a one to one correspondence among all these representations of a quantum state.

\subsection{Reconstruction of the wave function}

Reconstruction of $\psi(x)$ from the Wigner function

$$
\int W(x, p) \mathrm{e}^{-2 i p \xi^{\prime} / \hbar} d p=\int \psi^{*}(x+\xi) \delta\left(\xi-\xi^{\prime}\right) \psi(x-\xi) d \xi=\psi^{*}\left(x+\xi^{\prime}\right) \psi\left(x-\xi^{\prime}\right)
$$

Let us choose an $a$ such that

$$
\int W(a, p) d p=\psi^{*}(a) \psi\left(a^{\prime}\right) \neq 0
$$

and $\xi^{\prime}=a-x$,

$$
\begin{gathered}
\int W(x, p) \mathrm{e}^{-2 i p(a-x) / \hbar} d p=\psi^{*}(a) \psi(2 x-a) \\
2 x-a \rightarrow x \\
\psi(x)=\frac{1}{\psi^{*}(0)} \int W\left(\frac{x+a}{2}, p\right) \mathrm{e}^{i p(x-a) / \hbar} d p \\
\psi(x)=\frac{1}{\psi^{*}(0)} \int W\left(\frac{x}{2}, p\right) \mathrm{e}^{i p x / \hbar} d p
\end{gathered}
$$

Reconstruction of the Wigner function from the tomogram

$$
W(x, p)=\int \mathcal{W}(X, \mu, \nu) \mathrm{e}^{i(X-\mu x-\nu p)} d X d \mu d \nu
$$

consequently the recontruction of the wave function from the tomogram descends from the preceding equations, in the case $a=0$,

$$
\psi(x)=\frac{\hbar}{2 \pi} \frac{1}{\psi^{*}(0)} \int \mathcal{W}(X, \mu, \nu) \mathrm{e}^{i\left(X-\mu \frac{x}{2}-\nu p+\frac{p x}{\hbar}\right)} d X d \mu d \nu d p
$$

Integrating with respect to $p$ and $\nu$ we finally obtain

$$
\psi(x)=\frac{\hbar}{2 \pi} \frac{1}{\psi^{*}(0)} \int \mathcal{W}(X, \mu, \nu) \mathrm{e}^{\left.i\left(X-\frac{\mu x}{2}\right)\right)} d X d \mu .
$$




\subsection{Properties of the tomograms}

The symplectic tomogram $\mathcal{W}(X, \mu, \nu)$ has the properties which follow from its definition by using the known properties of delta function, namely,

1) Nonnegativity

$$
\mathcal{W}(X, \mu, \nu) \geq 0
$$

(this holds by observing that the trace of the product of two positive operators is a positive number).

2) Normalization

$$
\int \mathcal{W}(X, \mu, \nu) d X=1
$$

These first two conditions are important because the tomogram is a probability function.

3) homogeneity

$$
\mathcal{W}(\lambda X, \lambda \mu, \lambda \nu)=\frac{1}{|\lambda|} \mathcal{W}(X, \mu, \nu)
$$

\subsection{Stationary phase approximation for tomograms}

If we write the wave function in the quasi classical approximation, which corresponds to a stationary solution where the energy $E$ is a constant and the amplitude and the phase $S$ are connected. $S$ is a solution of the Hamilton-Jacobi equation.

$$
\psi(x)=|\psi(x)| \mathrm{e}^{i S(x)}
$$

in $|\psi(x)|$ varies slowly the tomogram is given in the following way,

$$
\mathcal{W}(X, \mu, \nu)=\frac{1}{2 \pi|\nu|}\left|\int\right| \psi(y)\left|\exp \left[i\left(S(y)+\frac{\mu}{2 \nu} y^{2}-\frac{X}{\nu} y\right)\right]\right|^{2}
$$

and applying the stationary phase approximation to obtain

$$
\mathcal{W}(X, \mu, \nu) \approx \frac{1}{2 \pi|\nu|}\left|\psi\left(x_{0}\right)\right|^{2}\left|\frac{\partial^{2} S}{\partial x^{2}}\right|_{x=x_{0}}+\left.\frac{\mu}{\nu}\right|^{-1}
$$

where $x_{0}$ is the solution of equation

$$
\frac{\partial S}{\partial x}+\frac{\mu}{\nu} x-\frac{X}{\nu}=0
$$

If eq. 2.33 has multiple roots $\left(x_{0}^{(1)} \ldots x_{0}^{(N)}\right)$ then the tomogram becomes 


$$
\mathcal{W}(X, \mu, \nu) \approx \frac{1}{2 \pi|\nu|}\left|\sum_{i=1}^{N} \frac{\psi\left(x_{0}^{(i)}\right)}{\sqrt{\left.\frac{\partial^{2} S}{\partial x^{2}}\right|_{x=x_{0}^{(i)}}+\frac{\mu}{\nu}}}\right|^{2} .
$$

We notice that

$$
\frac{\mu}{\nu} x-\frac{X}{\nu}=-p
$$

so eq. 2.33) expresses the condition of classical correlation,

$$
p=\frac{\partial S}{\partial x} .
$$

The stationary points, solutions of eq. 2.33 are they are the points where the integrand is maximum giving the most important contribution to the value of the integral. This means that the meaningful points which lead to the semiclassical solutions are at the peaks of the tomographic amplitudes, which lead to the solutions given in eq. 2.31.

\subsection{Classical tomograms}

The definition of tomogram 2.7 can be extended to any distribution on the phase space. Therefore we can extend this definition to classical distributions $f(q, p)$, e.g. solutions of the Boltzmann equation,

$$
\mathcal{W}(X, \mu, \nu)=\int f(x, p) \delta(X-\mu x-\nu p) d x d p .
$$

If the classical probability distribution $f(q, p)$ is normalized then also the tomogram is normalizedand satisfies the following conditions,

$$
\begin{gathered}
\int \mathcal{W}(X, \mu, \nu) d X=1 \\
\mathcal{W}(X, 1,0)=\int f(x, p) d p
\end{gathered}
$$

and

$$
\mathcal{W}(X, 0,1)=\int f(x, p) d x .
$$

For example taking the distribution function

$$
f(q, p)=\delta\left(x-x_{0}\right) \delta\left(p-p_{0}\right)
$$

introduced at the beginning of this section, we obtain by eq, 2.34 that the tomogram of a particle is

$$
\mathcal{W}(X, \mu, \nu)=\delta\left(X-\mu x_{0}-\nu p_{0}\right)
$$


For a moving particle the time dependent distribution function is

$$
f(q, p, t)=\delta(x-x(t) \delta(p-p(t))
$$

and the corresponding tomogram is

$$
\mathcal{W}(X, \mu, \nu)=\delta(X-\mu x(t)-\nu p(t) .
$$

In particular for a free particle with mass $m$ and given the equations of motion

$$
\begin{aligned}
& x(t)=x_{0}+\frac{p}{m} t \\
& p(t)=p_{0}=\text { const } .
\end{aligned}
$$

the particle describes a trajectory on the phase space given by the distribution function

$$
f(q, p, t)=\delta\left(x-x_{0}+\frac{p}{m} t\right) \delta\left(p-p_{0}\right)
$$

and the corresponding tomogram is

$$
\mathcal{W}(X, \mu, \nu)=\delta\left(X-\mu\left(x_{0}+\frac{p_{0}}{m} t\right)-\nu p_{0}\right) .
$$

\section{The De Sitter universe}

The de Sitter model was of crucial importance because it showed that the cosmological constant, contrary to what Einstein believed initially, makes the universe expand even in the absence of material fields. It is given by the equation

$$
G_{\mu \nu}=\Lambda g_{\mu \nu}
$$

abd the metric is the homogeneous and isotropic metric

$$
d s^{2}=N^{2} c^{2} d t^{2}-a^{2}(t)\left(\frac{d r^{2}}{1-k^{2} r^{2}}+r^{2}\left(d \theta^{2}+\sin ^{2} \theta d \phi^{2}\right)\right)
$$

where $N$ is the lapse, the function that encodes the choice of the time coordinate. If $k=0$ the classical solution gives an exponential expansion

$$
a(t) \propto e^{H t}
$$

where the Hubble constant $H$ is

$$
H=\sqrt{\frac{\Lambda}{3}}
$$

It must be noted that there is a singularity at $t=-\infty$, i.e. the cosmological constant violates the Hawking-Penrose conditions. 
The importance of the de Sitter model in cosmology was that it confirmed that General Relativity predicted a non static universe. This model was reintroduced with the inflationary paradigm to overcome the drawbacks of traditional cosmological models which were unable to explain in a natural way the flatness and the homogeneity of the universe.

Let us consider a closed homogeneous and isotropic model with a cosmological constant $\Lambda$ was used in quantum cosmology. Using the notations of [26][27] [31], we write the metric

$$
d s^{2}=l_{p}^{2}\left[-N^{2}(t) d t^{2}+a^{2}(t) d \Omega_{3}^{2}\right]
$$

where $a(t)$ is the expansion factor of the universe, $d \Omega_{3}^{2}$ is the metric of the unit three-sphere $N(t)$ is the lapse function, $l_{p}=2 / 3 \ell_{p}$, with $\ell_{p}$ the Planck length. Introducing the following variables

$$
q=a^{2} \quad \tilde{N}=N q
$$

the Hamiltonian takes the form

$$
\mathcal{H}=\frac{1}{2}\left(-4 p^{2}+\lambda q-1\right)
$$

where $\lambda$ is the cosmological constant in Planck units. The ADM formalism implies the constraint

$$
\mathcal{H}=0
$$

this corresponds to a trajectory on the phase space. At a fixed time the distribution on the phase space reduces to just one point of this trajectory. Differently from the example given in the previous section (see eq [2.38), position and momentum are not independent.

The equation of motion tells us how "fast" thie universe runs along this trajectory.

We represent this trajectory with the distribution

$$
f(q, p)=\delta\left(-4 p^{2}+\lambda q-1\right)
$$

and the corresponding classical tomogram is

$$
\begin{aligned}
\mathcal{W}(X, \mu, \nu) & =\int \delta\left(-4 p^{2}+\lambda q-1\right) \delta(X-\mu q-\nu p) d q d p \\
& =\frac{1}{2|\mu|} \frac{1}{\left|\sqrt{\frac{\lambda^{2} \nu^{2}}{16 \mu^{2}}+\frac{\lambda X}{\mu}-1}\right|} .
\end{aligned}
$$

This tomogram cannot be normalized as it it not integrable on $-\infty$ to $+\infty$. It can be normalized only if the function 3.10 has a compact support $C$. We the inferior value of the interval is giving by requiring the reality of the equare root,

$$
\frac{\lambda^{2} \nu^{2}}{16 \mu^{2}}+\frac{\lambda X}{\mu}-1 \geq 0
$$


i.e.

$$
X \geq \frac{\mu}{\lambda}\left(1-\frac{\lambda^{2} \nu^{2}}{16 \mu^{2}}\right)
$$

The superior iimit of $C$ follows by imposing the normalization of the tomogram 2.35.

$$
\int_{-\infty}^{\infty} \mathcal{W}(X, \mu, \nu)=\frac{1}{2|\mu|} \int_{\frac{\mu}{\lambda}\left(1-\frac{\lambda^{2} \nu^{2}}{16 \mu^{2}}\right)}^{\lambda \mu+\frac{\mu}{\lambda}\left(1-\frac{\lambda^{2} \nu^{2}}{16 \mu^{2}}\right)} \frac{1}{\left|\sqrt{\frac{\lambda^{2} \nu^{2}}{16 \mu^{2}}+\frac{\lambda X}{\mu}-1}\right|}=\frac{\mu}{|\mu|}
$$

which is equal to 1 only for positive $\mu$ In conclusion we redefine the function 3.10 , with support on the compact interval,

$$
\left[1-\frac{\lambda^{2} \nu^{2}}{16 \mu^{2}}, \lambda \mu+1-\frac{\lambda^{2} \nu^{2}}{16 \mu^{2}}\right] .
$$

as

$$
\mathcal{W}(X, \mu, \nu)= \begin{cases}0 & X<1-\frac{\lambda^{2} \nu^{2}}{16 \mu^{2}} \\ \frac{1}{2|\mu|} \frac{1}{\mid \sqrt{\frac{\lambda^{2} \nu^{2}}{16 \mu^{2}}+\frac{\lambda X}{\mu}-1 \mid}} & 1-\frac{\lambda \mu-\nu^{2}}{16 \mu^{2}} \leq X \leq \lambda \mu+1-\frac{\lambda^{2} \nu^{2}}{16 \mu^{2}} \\ 0 & X>\lambda \mu+1-\frac{\lambda^{2} \nu^{2}}{16 \mu^{2}}\end{cases}
$$

with $\mu>0$.

Let us assume that the cosmological constant has the value currently accepted. In Planck units $\lambda=5.6 \cdot 10^{-122} t_{p}^{-2}$, therefore to all practical effects we can consider the function (3.15) as a Dirac delta. We can show this by considering for sake of simplicity the integral of any function $f(x)$ with the function $1 /\left(2 c \sqrt{x-x_{0}}\right)$ with support on the closed interval $\left[x_{0}, x_{0}+c^{2}\right]$

$$
\begin{aligned}
\frac{1}{2 c} \int_{x_{0}}^{x_{0}+c^{2}} \frac{f(x)}{\sqrt{c x-x_{0}}} d x & =\frac{1}{2 c} \int_{x_{0}}^{x_{0}+c^{2}}\left(\frac{f\left(x_{0}\right)}{\sqrt{x-x_{0}}}+\frac{f^{\prime}\left(x_{0}\right)}{\sqrt{x-x_{0}}}\left(x-x_{0}\right)+\ldots\right) d x \\
& =\frac{1}{2 c}\left(\left.2 f\left(x_{0}\right) \sqrt{x-x_{0}}\right|_{x_{0}} ^{x_{0}+c^{2}}+\left.f^{\prime}\left(x_{0}\right)\left(x-x_{0}\right)^{3 / 2}\right|_{x_{0}} ^{x_{0}+c^{2}}+\ldots\right) \\
& =f\left(x_{0}\right)+f^{\prime}\left(x_{0}\right) \times c^{2}+O\left(c^{4}\right)
\end{aligned}
$$

if $c$ is negligible integration (3.16) gives approximately $f\left(x_{0}\right)$.

In conclusion, if the cosmological constant is of the order of magnitude of the value accepted nowadays the classical tomogram of a de Sitter universe may be expressed by a delta function, 


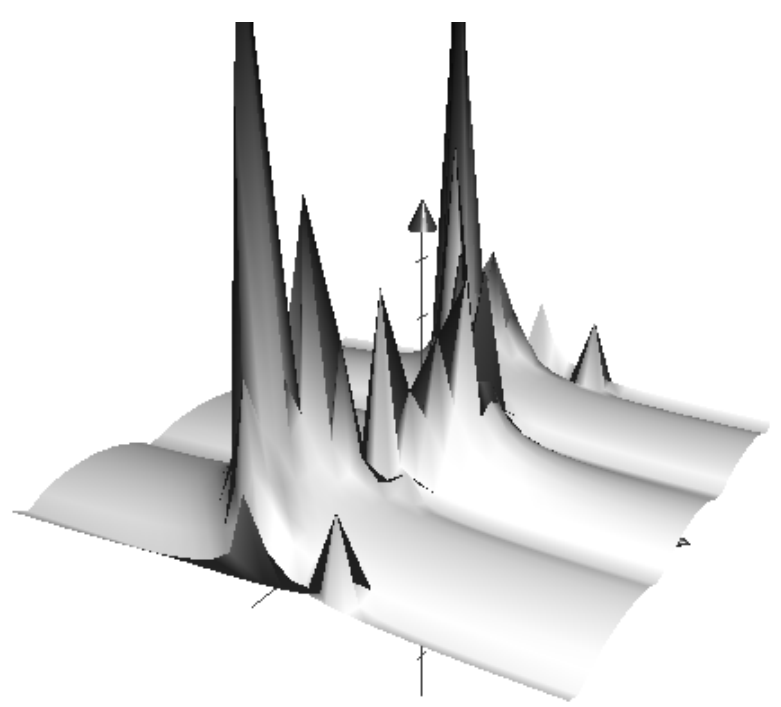

Figure 1: The tomogram of the classical de Sitter universe

$$
\mathcal{W}(X, \mu, \nu)=\delta\left(\frac{\lambda}{\mu} X+\frac{\lambda^{2} \nu^{2}}{16 \mu^{2}}-1\right) .
$$

It is interesting to note that in the classical universe the sharp conditions of sharp homogeneity are determined by the extreme smallness of the cosmological constant.

\section{The de Sitter quantum cosmological mod- els}

In quantum cosmology the Hamiltonian constraint becomes an equation, called the Wheeler De Witt equation, in which the Hamiltonian operator annihilates the wave function $\psi(q)$

$$
\hat{\mathcal{H}} \psi(q)=0 .
$$

The wave function represents the state of the quantum universe. 
The Wheeler-DeWitt equation in the de Sitter universe is

$$
\left(4 \hbar^{2} \frac{d^{2}}{d q^{2}}+\lambda q-1\right) \psi(q)=0
$$

The properties of this equation were discussed in [25]. With the change of variables

$$
x=-\left(\frac{\lambda}{4 \hbar^{2}}\right)^{1 / 3}\left(q-\frac{1}{\lambda}\right)=\frac{1-\lambda q}{(2 \hbar \lambda)^{2 / 3}}
$$

equation 4.2 becomes the Airy equation

$$
\frac{d^{2} \psi(x)}{d x^{2}}-x \psi(x)=0
$$

whose solutions are expressed by the integral on the complex plane

$$
\psi(x)=\int_{C} \exp \left(x z-\frac{z^{3}}{3}\right)
$$

and depend on the choice of the path $C$ [29] [30].

There are has two independent solutions. The solutions of interest are those which take real values. These are the $\operatorname{Ai}(x)$ defined by taking as path $C$ the imaginary axis from $-i \infty$ to $+i \infty$ so that its integral representation is

$$
\operatorname{Ai}(x)=\frac{1}{2 \pi} \int_{-\infty}^{+\infty} \exp \left[i\left(\frac{z^{3}}{3}+x z\right)\right] d z
$$

and $\operatorname{Bi}(x)$ which is is obtained taking the path $C$ following the imaginary axis from $+i \infty$ to 0 and then along the real axis from 0 to $+\infty$, its integral representation is

$$
\operatorname{Bi}(x)=\frac{1}{\pi} \int_{0}^{+\infty}\left[\exp \left(-\frac{z^{3}}{3}+x z\right)+\sin \left(\frac{z^{3}}{3}+x z\right)\right] d z .
$$

$\operatorname{Bi}(x)$ has the property to be real valued for real $x$. Any other solution of the Airy equation is given by a linear combination of these two functions.

Among all the solutions we can select the physically meaningful ones by imposing appropriate initial conditions. Of particular interest are the conditions which predict a classical evolution with the characteristics observed in the present universe.

In this context, among the various initial conditions proposed the best known and most studied are the Hartle and Hawking "no-boundary condition" and Vilenkin's "tunneling of the universe from nothing beginning " which was resubmitted in a different form by Linde.

With the initial no-boundary solution, Hartle and Hawking have intended to restrict the set of solutions of the Wheeler De Witt equation 
by selecting the set of 4-geometries allowed such that there is not a singular initial boundary differently from the classical solutions. This is obtained by "smoothing the geometry of the universe off in an imaginary time". 33. For example, whereas a surface with $\sqrt{h}=0$ (with $h$ the determinant of the spatial metric) would be singular in a Lorentzian signature metric, this is not necessarily the case if the metric is of Euclidean signature. In other words it is necessary to choose the initial condition ensuring the closure of the four-geometry 34]

Vilenkin represented with the tunneling of universe an initial state of the universe in an analogy with tunneling effect in quantum mechanics.

As in quantum mechanics the solutions contain "ingoing" and "outgoing" modes, in quantum cosmology one has to contracting and expanding modes. According yo Vilenkin's proposal the universe wave function consists of those containing only expanding modes at the parts of the boundary of superspace corresponding to the classical singular four-geometries. Moreover a regularity condition, that $\psi$ be everywhere bounded, is also imposed. 34

In this paper we consider the different wave functions In a de Sitter minisuperspace. We reproduce the results obtained in [25] and extend the discussion of these models to the Wigner functions (see [26]|27] and [31] ) and to the tomographic representation.

\subsection{Wave Functions}

In the de Sitter quantum cosmological models the initial conditions are linear combinations of the Airy functions. The form of the wave functions corresponding to the initial conditions was obtained by Halliwell and Louko in [25]. It particular they found that the Hartle and Hawking wave function is

$$
\psi_{H H}=A \operatorname{Ai}\left(\frac{1-\lambda q}{(2 \hbar \lambda)^{2 / 3}}\right),
$$

Vilenkon's tunneling from nothing is obtained by the complex combination

$$
\psi_{V}\left(\frac{1-\lambda q}{(2 \hbar \lambda)^{2 / 3}}\right)=\frac{B}{2}\left(\operatorname{Ai}\left(\frac{1-\lambda q}{(2 \hbar \lambda)^{2 / 3}}\right)+i \operatorname{Bi}\left(\frac{1-\lambda q}{(2 \hbar \lambda)^{2 / 3}}\right)\right)
$$

and finally Linde's condition is given by

$$
\psi_{L}=-i C \cdot \operatorname{Bi}\left(\frac{1-\lambda q}{(2 \hbar \lambda)^{2 / 3}}\right)
$$

Where $A, B$ and $C$ are normalization constants which can be determined. 
The properties of these different wave functions can be better highlighted if we consider the following approximate forms at large values of $q$ (see [30]),

$$
\begin{gathered}
\psi_{H H} \approx \frac{(2 \lambda \hbar)^{1 / 6}}{2\left(\pi^{2}(\lambda q-1)\right)^{1 / 4}}\left(\mathrm{e}^{i S}+\mathrm{e}^{-i S}\right) \\
\psi_{V} \approx i \frac{(2 \lambda \hbar)^{1 / 6}}{2\left(\pi^{2}(\lambda q-1)\right)^{1 / 4}} \mathrm{e}^{-i S} \\
\psi_{L} \approx i \frac{(2 \lambda \hbar)^{1 / 6}}{2\left(\pi^{2}(\lambda q-1)\right)^{1 / 4}}\left(\mathrm{e}^{i S}-\mathrm{e}^{-i S}\right)
\end{gathered}
$$

where

$$
S=\frac{1}{3 \lambda \hbar}(\lambda q-1)^{3 / 2}-\frac{\pi}{4}
$$

Eqs. (4.11)- (4.13) show that the Vilenkin solution actually contains only the expanding modes, while the Hartle and Hawking and the Linde solutions contain both contracting and expanding modes. This behavior is most emphasized when one introduces the functions of Wigner and the tomograms, where the superposition of modes shows the presence of positive and negative interferences in the phase space. For this reason it is interesting to discuss the properties of these functions in relation to the initial conditions. In this way it will be possible to evaluate the major and minor probabilities of evolution of the universe. In the following we will summarize the results obtained for the Wigner functions and their properties.

\subsection{Wigner Functions}

The Wigner function was introduced by Wigner in his paper "On The Quantum Correction For Thermodynamic Equilibrium"[20] after observing that in quantum mechanics there was not a simple expression for probability as in classical statistical mechanics. As a consequence of the impossibility to measure simiultaneously the position and the momenta, Wigner functions may take negative values. Therefore they cannot be interpreted as probability functions.

In quantum cosmology Wigner functions were introduced because, although the formalism in phase space completely describes all the aspects of quantum mechanics, the strength of formalism is its ability of dealing more clearly with semiclassical problems and the transition from quantum into classical systems.

Firstly because as noted by Wheeler, the wave function is not suitable for implementing a classic limit because it is not localizable and has multiple peaks. 
Secondly, it has been hypothesized by some researchers that the presence in a semiclassical model of a correlation between the canonical variables is a signature of a classical transition

Finally, it can be established that in quantum cosmology there can not be a classical transition without quantum decoherence and it has been pointed out by various authors that Wigner's functions proves suitable for studying it.

Often quantum decoherence it is identified with the cancellation of the interference terms typical of the quantum phenomena. But it the result of a coarse graining of the quantum system. It can be achieved in different ways. For example if the relevant measures can be done only by specifying the variables $q^{i}$ not at all times $t$, but in a discrete number of times $t_{i}$ with $i=1,2,3, \ldots$, otherwise specifying not all the variables $q^{i}$ at any one time, but some of them or finally by not specifying the definite values of the $q^{i}$, but only range of values $\Delta^{i}$ they can assume 24.

On the other side the quantum decoherence manifests itself in open systems through their interaction with an external environment, similarly in quantum cosmology the same is obtained by implementing a coarse graining obtained through a trace-over unobservables.

Some authors tried to find particular solutions of the Wheeler-DeWitt equation peacke araound a subset of solution of the field equation, so that the boundary conditions of the Wheeler-DeWitt equation lead, in the classical limit, to the initial conditions on the classical solutions, see for example 22]. To show this an approximate solution was used to construct the Wigner function. That this procedure was incorrect was shown subsequently in [23], where it was shown that the Wigner functions obtained with approximate functions and do not retain the same properties of those obtained with the complete solutions as it happens for example with the harmonic oscillator.

The exact solutions for a de Sitter quantum cosmological model was obtained by[26]|27] using the results obtained by Berry [28]. Which can be obtained also by applying the 2.2 tp the wave functions obtained by Halliwell and Louko in [25]. Finally in [31 a complete analysis of the Wigner function from the point of view of the Deformed Quantum Cosmology. It was shown that the previous solutions can be obtained from the Moyal equations(for an overview of the definition of the star product and the Moyal equation see [32]).

$$
\mathcal{H} \star W(q, p)=0
$$

The Hartle-Hawking Wigner function can be derived directly from 4.15 or by applying eq. (2.2) to the wave function (4.8) It takes the form,

$$
W_{H H}(q, p)=\frac{2^{1 / 3} A^{2}}{\pi(\hbar \lambda)^{1 / 3}} \mathrm{Ai}\left[\frac{4 p^{2}-\lambda q+1}{(\hbar \lambda)^{2 / 3}}\right] .
$$

Only in this case it is possible to obtain explictly this Wigner function. 
In the other two cases the wave function contains the function $\operatorname{Bi}(q, p)$ which is not a limited function, even if formally it is a solution of the Moyal equation. It must be excluded because the Wigner function $\mathrm{s}$ to be normal or normalizable (see eq. 2.3).

\section{The quantum tomograms of the universe and the classical limit}

In this section we will stress the validity and effectiveness of the tomographic approach by discussing the properties of the initial HartleHawking conditions of which we have an analytical expression.

Let us derive the tomogram corresponding to the wave function 4.8 . By inserting (4.8) in 2.10) we immediately obtain

$$
\mathcal{W}(X, \mu, \nu)=\frac{A^{2}}{|\mu|}\left|\operatorname{Ai}\left(\frac{1}{(2 \hbar \lambda)^{2 / 3}}\left(1-\frac{\lambda X}{\mu}-\frac{\lambda^{2}}{16} \frac{\nu^{2}}{\mu^{2}}\right)\right)\right|^{2}
$$

where the constant $A$ will be determined using the asymptotic expression of the tomogram in a similar way to the procedure described section 21 of [39] for the one variable wave fucntions. The wave function is formally the same found for an electrion subject to a linear potential or a constant electric field (see [39]): In [40] the properties of the wave function were discussed and the corresponding Wigner function and tomogram were obtained. We illustrate the qualitative properties of the tomogram in figure, we put $\lambda=1 / 2$ such that $2 \hbar \lambda=1$. We choose the parametrization $\mu=\cos \phi$ and $\nu=\sin \phi$ (typically use for optical tomograms) so that the tomogram could be plotted in the variables $X$ and $\phi$. The graphic of the tomogram is given in figure 2

The determination of the quantum tomogram allows us to study the classical limit. One of the ways to do this is to determine the limit of the tomogram for $\hbar \rightarrow 0$ and compare the result with the classical tomogram (3.10). This problem was addressed in [41.

Let us put

$$
\psi(x)=\hbar^{\gamma / 2} \psi(\hbar x)
$$

so that its norm is invariant. Then we write the tomogram constructed with [5.2]

$$
\mathcal{W}(X, \mu, \nu)=\frac{1}{2 \pi \hbar|\nu|}\left|\int \hbar^{\gamma / 2} \psi\left(\hbar^{\gamma} y\right) \exp \left[i\left(\frac{\mu}{2 \hbar \nu} y^{2}-\frac{X}{\hbar \nu} y\right)\right]\right|^{2} d y
$$

to calculate the limit $\hbar \rightarrow 0$ we apply the delta theorem which states that for any function $f(x)$ such that

$$
\int_{-\infty}^{\infty} f(x)=N
$$




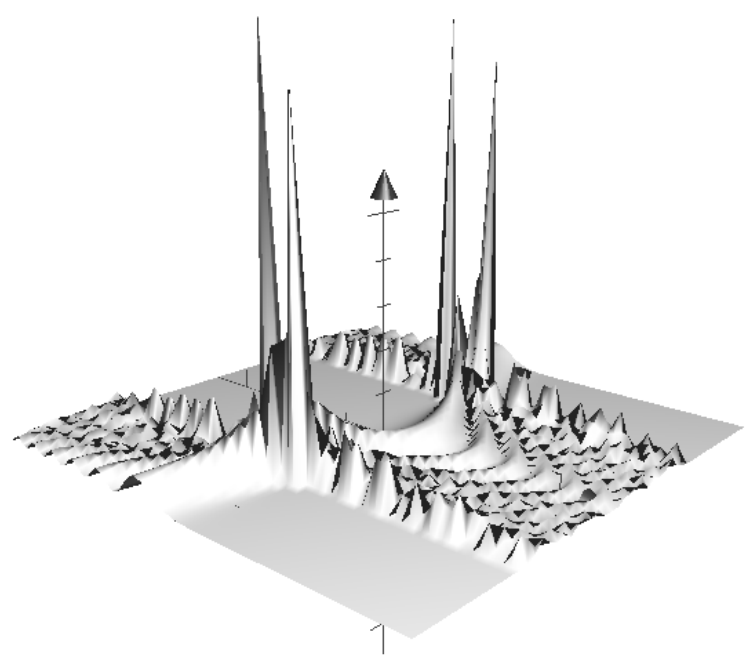

Figure 2: The rich structure of the Hartle Hawking tomogram

we can show that it has the limit

$$
\lim _{n \rightarrow \infty} n f\left(n\left(x-x_{0}\right)\right)=N \delta\left(x-x_{0}\right) .
$$

In [30] this theorem is discussed and demostrated in a different way for the Airy function $A i(x)$.

Let us apply this theorem to the Wigner function 4.16 by considering the limit $(2 \hbar \lambda)^{2 / 3} \rightarrow 0$ With an appropriate choice of constant $\mathrm{A}$ it follows that

$$
\lim _{(2 \hbar \lambda)^{2 / 3} \rightarrow 0} W_{H H}(q, p)=\delta\left(-4 p^{2}+\lambda q-1\right)
$$

We conclude that from the point of view of the Wigner functions the Hartle and Hawking initial condition has a classical limit.

Let us now examine the transition from quantum to classical from a tomographic point of view.

From [5.1] we see that the limit $(2 \hbar \lambda)^{2 / 3} \rightarrow 0$ implies that it is sufficient to use the asymptotic expressions of the Airy function $\operatorname{Ai}(x)$. They are

$$
\operatorname{Ai}(x) \approx \frac{1}{2 \pi^{1 / 2}|x|^{1 / 4}} \mathrm{e}^{-\xi} L(-\xi)
$$


and

$$
\operatorname{Ai}(-x) \approx \frac{1}{\pi^{1 / 2}|x|^{1 / 4}}\left[\sin \left(\xi-\frac{\pi}{4}\right) Q(\xi)+\cos \left(\xi-\frac{\pi}{4}\right) P(\xi)\right]
$$

where the functions $L(\xi), P(\xi)$ and $Q(\xi)$ are given by the following series,

$$
\begin{gathered}
L(\xi)=\sum_{s=0}^{\infty} \frac{a_{s}}{\xi^{s}}=1+\frac{3 \cdot 5}{1 ! 216} \frac{1}{\xi}+\frac{5 \cdot 7 \cdot 9 \cdot 11}{2 ! 216^{2}} \frac{1}{\xi^{2}}+\ldots \\
P(\xi)=\sum_{s=0}^{\infty}(-1)^{s} \frac{a_{2 s}}{\xi^{2 s}}=1-\frac{5 \cdot 7 \cdot 9 \cdot 11}{2 ! 216^{2}} \cdot \frac{1}{\xi^{2}}+\frac{9 \cdot 11 \cdot 13 \cdot 15 \cdot 17 \cdot 19 \cdot 21 \cdot 23}{4 ! 216^{4}} \cdot \frac{1}{\xi^{4}}+\ldots
\end{gathered}
$$

and

$$
Q(\xi)=\sum_{s=0}^{\infty}(-1)^{s} \frac{a_{2 s+1}}{x^{2 s+1}}=\frac{3 \cdot 5}{1 ! 216} \frac{1}{\xi}-\frac{7 \cdot 9 \cdot 11 \cdot 13 \cdot 15 \cdot 17}{3 ! 216^{3}} \frac{1}{\xi^{3}}+\ldots,
$$

where $a_{s}=\frac{\Gamma\left(3 s+\frac{1}{2}\right)}{54^{s} s ! \Gamma\left(s+\frac{1}{2}\right)}$ and $\xi=\frac{2}{3} S^{3 / 2}$. In this approximation when the argument is negative the tomogram is

$$
\begin{aligned}
\mathcal{W}(X, \mu, \nu) & \approx \frac{A^{2}}{8 \pi^{2} \hbar|\mu|} \frac{(2 \hbar \lambda)^{4 / 3}}{\left|1-\frac{\lambda X}{\mu}-\frac{\lambda^{2}}{16} \frac{\nu^{2}}{\mu^{2}}\right|^{1 / 2}} \\
& \times\left|P(S) \cdot \cos \left(\frac{2}{3} S^{3 / 2}-\frac{\pi}{4}\right)+Q(S) \cdot \sin \left(\frac{2}{3} S^{3 / 2}-\frac{\pi}{4}\right)\right|^{2} x, .
\end{aligned}
$$

When the argument is positive the approximated tomogram has the form

$$
\begin{gathered}
\mathcal{W}(X, \mu, \nu) \approx \frac{A^{2}}{8 \pi^{2} \hbar|\mu|} \frac{(2 \hbar \lambda)^{4 / 3}}{\left|1-\frac{\lambda X}{\mu}-\frac{\lambda^{2}}{16} \frac{\nu^{2}}{\mu^{2}}\right|^{1 / 2}}\left|\mathrm{e}^{-S} L(-S)\right|^{2} \\
S=\frac{1}{(2 \hbar \lambda)^{2 / 3}}\left(1-\frac{\lambda X}{\mu}-\frac{\lambda^{2}}{16} \frac{\nu^{2}}{\mu^{2}}\right)
\end{gathered}
$$

The normalization constant $A$ can be fixed by requiring that the quantum and classical tomograms have the same coefficient, i.e.

$$
A=\frac{2^{5 / 6} \pi}{\hbar^{1 / 6} \lambda^{2 / 3}} .
$$

Let us determine the limit of the functions 5.12 and 5.13 for $(2 \hbar \lambda)^{2 / 3} \rightarrow$ 0 . First we notice that righthand side of eq. (5.13) is zero because

$$
\lim _{(2 \hbar \lambda)^{2 / 3} \rightarrow 0} \mathrm{e}^{-2 S}=0 .
$$




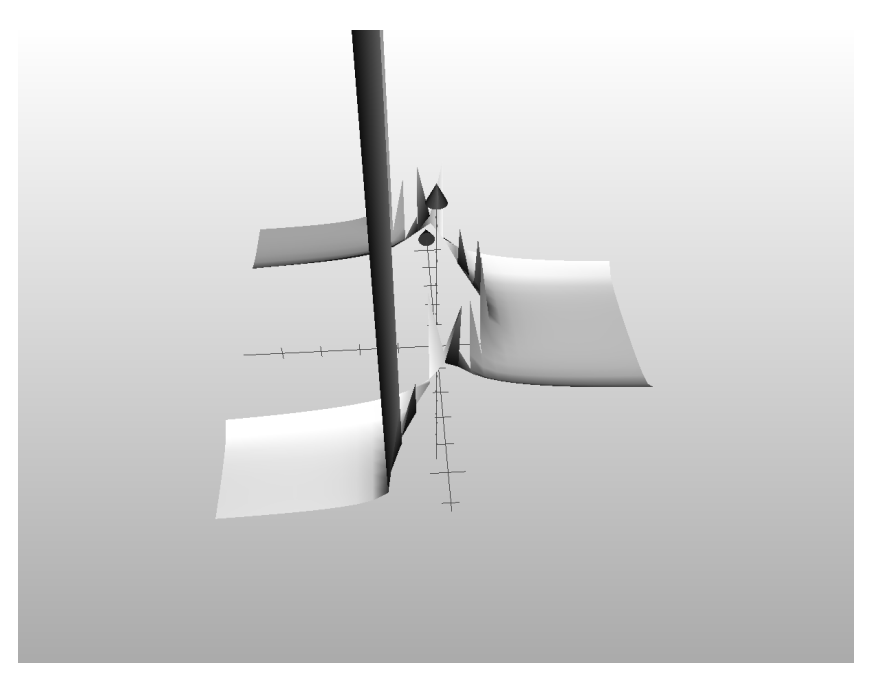

Figure 3: The classical tomogram

There is no contribution to the tomogram from the positive sector of the Airy function. Let us study the negative sector of $\operatorname{Ai}(S)$. From eqs. (5.10) and [5.11) we see that

$$
\lim _{(2 \hbar \lambda)^{2 / 3} \rightarrow 0} Q(S)=0
$$

and

$$
\lim _{(2 \hbar \lambda)^{2 / 3} \rightarrow 0} P(S)=1,
$$

then in eq.5.12) we are left with the square modulus of the cosine, but its argument goes to infinity and the quantum tomogram does not converge to the classical tomogram, even if one of its factors coincides with the expression (3.10).

Even modifying the expression of the tomogram as in [5.3) to apply the delta theorem, we do not obtain the classical limit. For example if one adjusts the exponent $\gamma$ of $\hbar$ such that the cosine and the sine go a to a constant, the functions $P$ and $Q$ will take both an infinite value. Considering that it is not possible to determine a finite tomogram for the Vilenkin and Linde wave functions, we conclude that none of the proposed initial conditions lead to a classical universe.

The following tomogram

$$
\begin{aligned}
\mathcal{W}(X, \mu, \nu) & =\frac{A^{2}}{|\mu|} \mid \frac{1}{2}\left(\operatorname{Ai}\left(\frac{1}{(2 \hbar \lambda)^{2 / 3}}\left(1-\frac{\lambda X}{\mu}-\frac{\lambda^{2}}{16} \frac{\nu^{2}}{\mu^{2}}\right)\right)\right. \\
& \left.+i \operatorname{Bi}\left(\frac{1}{(2 \hbar \lambda)^{2 / 3}}\left(1-\frac{\lambda X}{\mu}-\frac{\lambda^{2}}{16} \frac{\nu^{2}}{\mu^{2}}\right)\right)\right)\left.\right|^{2}
\end{aligned}
$$




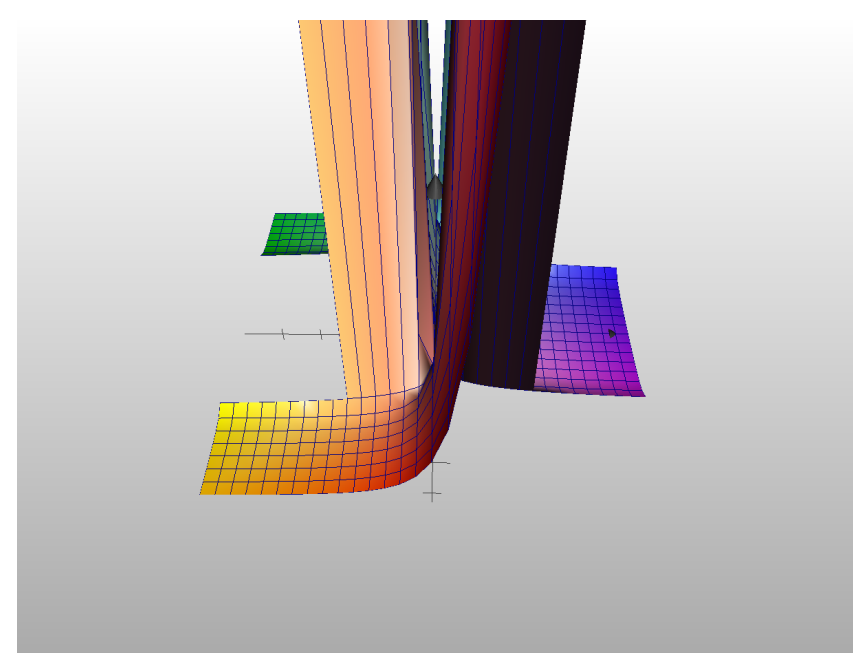

Figure 4: The quantum de Sitter tomogram

witnnh $A$ given by (5.15), provided the condition (2.25) is satisfied is a possbile solution because in the limit $(2 \hbar \lambda)^{2 / 3} \rightarrow 0$ its asymptotic expression reduces to the classical tomogram .

In this case the classical and quantum tomograms are compared in figures 3 and 4 to show that they can be superimposed see fig. 5 .

\section{Perspectives and conclusions}

The introduction of the tomographic analysis in quantum cosmology has enabled us to highlight some important properties of the solutions of the Wheeler DeWitt equation.

The main result of this paper is the study of the classical limit of a quantum universe is dominated by the cosmological constant and comparing it with the tomogram derived from the de Sitter classical solution. Although it is an extremely simple model, it still provides us with a very interesting result.

Indeed as the classical limit is achieved by taking the limit $(2 \hbar \lambda)^{2 / 3} \rightarrow$ 0 in eqs. (5.6) and [5.7), we can see that this limit is not necessarily obtained $\hbar \rightarrow 0$. Rather it looks like that the quantum-classical transition of the universe depends on the decay of the cosmological constant from values close to $\lambda \approx 1$ to the present value $\lambda_{c}=5.6 \cdot 10^{-122} t_{p}^{-2}$. Moreover it results that the state of the classical universe is constrained by the interval (3.14) yielding a very high degree of homogeneity.

Therefore the problem of the value of the cosmological constant 42] becomes crucial to understand the current large-scale structure of the universe. We noticed that even if the the Hartle-Hawking Wigner func- 


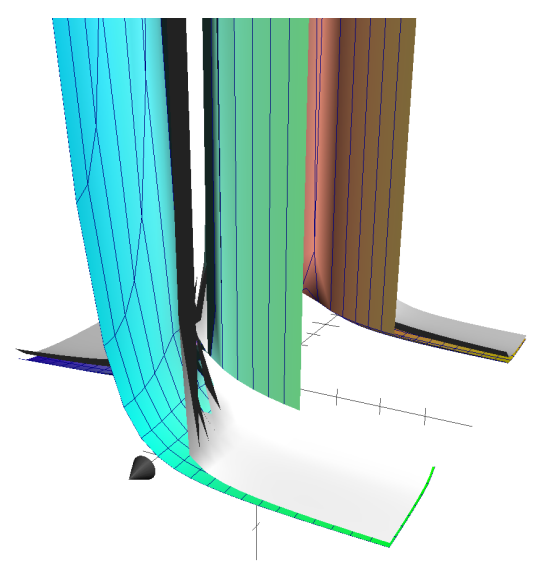

Figure 5: The superposition of the classical and quantum de Sitter tomogram

tion converges to the classical solution, its corresponding tomogram does not converge because when $(2 \hbar \lambda)^{2 / 3} \rightarrow 0$ its asymptotic expression oscillates rapidly, therefore it has not a limit.

However, we can not rule out that the universe can be described by this particular model. Since the final value of the cosmological constant, although small is different from zero, it could be that the limit of the Hartle and Hawking model describes equally well a universe dominated by the cosmological constant in which the material sources are negligible and where the galaxies can behave as test bodies. Since the integration interval should be of the same order of magnitude as the classical model, the tomogram would present some oscillations that would distinguish it from the classical model [3.10). An analysis of the distribution of motion of galaxies could falsify one of the two models or even both.

The second interesting point is that the normalization condition 2.35 restricts the range of values that $X$ can take. This suggests that a further investigation is needed to determine domain of the functions (4.9)4.10) such that the resulting tomogram can be expressed, even if in an analytic way.

On the other side according to the properties of the Airy functions, we introduced the tomogram (5.16) as a possible solution that converges to the classical solution [3.10). But the asymptotic expression of the oscillating part of the Airy functions converges to the inverse square 
root of $|-\xi|$, and the classical solution is inverse of the modulus of the square root of $\xi$. These two functions are not equal everywhere. The two tomograms can be superimposed only when

$$
\xi=\frac{\lambda^{2} \nu^{2}}{16 \mu^{2}}+\frac{\lambda X}{\mu}-1 \geq 0
$$

where the physical relevant regions of definition for $X$ can be found.

Of course the results of this paper cannot be considered conclusive, it will be necessary to study more general models taking in account the presence of scalar fields and more general material sources. The method introduced in this paper can be extended to many models in the vast literature of quantum cosmology from the traditional solutions of the Wheeler De Witt equation to the loop quantum cosmology [43] and for the further developments (see for example [44]).

Finally the tomographic approach can be extended beyond the analysis of the single wave functions of the universe, because the task of quantum tomography is the reconstruction of the initial state of a physical system starting from the experimental data, so with this perspective further work will be devoted to the phenomenological construction of the tomograms of the classical universe.

\section{References}

[1] R. L. Arnowitt, S. Deser and C. W. Misner, Gen. Rel. Grav. 40 (2008) 1997 doi:10.1007/s10714-008-0661-1 |gr-qc/0405109|.

[2] J. B. Hartle, In *Nishinomiya 1996, Physics in the 21st century* 179-199 |gr-qc/9701022.

[3] A. Fedorov, Phys. Lett. A 377 (2013) 2320.

[4] K. Vogel and H. Risken, Phys. Rev. A 40 (1989) 2847.

[5] Bertrand, J. and Bertrand, P. Found Phys (1987) 17, 397.

[6] G. Breitenbach and S. Schiller Homodyne tomography of classical and non-classical light, Journal of Modern Optics, 44 (1997) , 2207-2225

[7] S Mancini, V. I. Man'ko and P Tombesi 1995 Guantum Semiclass. Opt. 7615

[8] U. Janicke and M. Wilkens Journal of Modern Optics 42 (1995), 2183

[9] M. G. Raymer, M. Beck, and D. McAlister Phys. Rev. Lett. 721137 (1994) 
[10] Ch. Kurtsiefer, T. Pfau and J. Mlynek, Nature 386, 150 (1997)

[11] Ch, Kurtsiefer, J. Mlynek Appl. Phys. B 64, 85-90 (1997)

[12] D. F. Styer et al. American Journal of Physics 2002 70, 288-297 (2002)

[13] V. I. Manko, G. Marmo and C. Stornaiolo, "Radon transform of Wheeler-de Witt equation and tomography of quantum states of the universe," Gen. Rel. Grav. 37 (2005) 99 |gr-qc/0307084|.

[14] V. I. Man'ko, G. Marmo and C. Stornaiolo, "Cosmological dynamics in tomographic probability representation," Gen. Rel. Grav. 37 (2005) 2003 |gr-qc/0412091|.

[15] V. I. Man'ko, G. Marmo and C. Stornaiolo, "Tomographic entropy and cosmology," Gen. Rel. Grav. 40 (2008) 1449 [gr-qc/0612073].

[16] S. Capozziello, V. I. Man'ko, G. Marmo and C. Stornaiolo, "Tomographic Representation of Minisuperspace Quantum Cosmology and Noether Symmetries," Gen. Rel. Grav. 40 (2008) 2627 arXiv:0706.3018 [gr-qc]].

[17] S. Capozziello, V. I. Man'ko, G. Marmo and C. Stornaiolo, "A Tomographic description for classical and quantum cosmological perturbations," Phys. Scripta 80 (2009) 045901 |arXiv:0905.1244 [gr-qc]].

[18] C. Stornaiolo, "Tomographic Represention of Quantum and Classical Cosmology,” Astrophys. Space Sci. Proc. 38 (2014) 3, 211.

[19] C. Stornaiolo, “Tomographic cosmology,” Phys. Scripta 90 (2015) 7, 074032.

[20] E.P. Wigner, Phys. Rev. 40 (1932) 749.

[21] H. Grönewold, Physica 12 (1946) 405.

[22] J. J. Halliwell, Phys. Rev. D 36 (1987) 3626.

[23] A. Anderson, Phys. Rev. D 42 (1990) 585.

[24] J. B. Hartle, In *Jerusalem 1989, Proceedings, Quantum cosmology and baby universes* 65-157.

[25] J. J. Halliwell and J. Louko, Phys. Rev. D 39 (1989) 2206.

[26] S. Habib, Phys. Rev. D 42 (1990) 2566.

[27] S. Habib and R. Laflamme, Phys. Rev. D 42 (1990) 4056.

[28] Berry, M V, Phil. Trans. R. Soc. A. 287, (1977) 237-71. 
[29] Abramowitz, M. and Stegun, I. (1965). Handbook of Mathematical Functions, Dover Publications, New York.

[30] O. Vallee, M. Soares, Airy Functions and Applications to Physics, Imperial College Press, (2004)

[31] R. Cordero, H. Garcia-Compean and F. J. Turrubiates, Phys. Rev. D 83 (2011) 125030 doi:10.1103/PhysRevD.83.125030 arXiv: 1102.4379 [hep-th]].

[32] . C. K. Zachos, D. B. Fairlie and T. L. Curtright eds, Quantum Mechanics in Phase Space. An overview with selected papers World Scientific New Jersey, London, Singapore (2005)

[33] D. L. Wiltshire, "An Introduction to quantum cosmology," grqc/0101003.

[34] J. J. Halliwell, In *Jerusalem 1989, Proceedings, Quantum cosmology and baby universes* 159-243 and MIT Cambridge - CTP-1845 (90,rec.May) 108 p arXiv:0909.2566 [gr-qc]].

[35] A. I. Lvovsky and M. G. Raymer, Rev. Mod. Phys. 81 (2009) 299.

[36] Yuen H. P., and Chan V. W. S., , Optics Lett., (1983) 18, 177

[37] D. P. DiVincenzo, Fortschritte der Physik 48, 771 (2000).

[38] Robin Blume-Kohout New Journal of Physics, 12, (2010) 043034

[39] L. D. Landau and E. M. Lifshits, "Quantum Mechanics : NonRelativistic Theory,"

[40] Manko, V.I., Shchukin, E.V.: J. Russ. Laser Res. 22, 545 (2001)

[41] V.I. Man'ko, G. Marmo, A. Simoni, A. Stern, F. Ventriglia Physics Letters A 343 251-266 (2005)

[42] S. Weinberg, Rev. Mod. Phys. 61 (1989) 1.

[43] M. Bojowald, Living Rev. Rel. 11 (2008) 4.

[44] M. Bojowald, Rept. Prog. Phys. 78 (2015) 023901 